APS

physics

This is the accepted manuscript made available via CHORUS. The article has been published as:

\title{
Gravitational waveforms for neutron star binaries from binary black hole simulations
}

Kevin Barkett, Mark A. Scheel, Roland Haas, Christian D. Ott, Sebastiano Bernuzzi, Duncan A. Brown, Béla Szilágyi, Jeffrey D. Kaplan, Jonas Lippuner, Curran D. Muhlberger,

Francois Foucart, and Matthew D. Duez

Phys. Rev. D 93, 044064 - Published 24 February 2016

DOI: 10.1103/PhysRevD.93.044064 


\title{
Gravitational waveforms for neutron star binaries from binary black hole simulations
}

\author{
Kevin Barkett, ${ }^{1}$ Mark A. Scheel,${ }^{1}$ Roland Haas,${ }^{2,1}$ Christian D. Ott,${ }^{1}$ Sebastiano \\ Bernuzzi, ${ }^{1,3}$ Duncan A. Brown, ${ }^{4}$ Béla Szilágyi, ${ }^{5}$ Jeffrey D. Kaplan, ${ }^{1}$ Jonas \\ Lippuner, ${ }^{1}$ Curran D. Muhlberger, ${ }^{6}$ Francois Foucart, ${ }^{7,8}$ and Matthew D. Duez ${ }^{9}$ \\ ${ }^{1}$ TAPIR, Walter Burke Institute for Theoretical Physics, California Institute of Technology, Pasadena, CA 91125, USA \\ ${ }^{2}$ Albert Einstein Institute, Max-Planck-Institut für Gravitationsphysik, Potsdam, Germany \\ ${ }^{3}$ DiFeST, University of Parma, and INFN Parma I-43124 Parma, Italy \\ ${ }^{4}$ Department of Physics, Syracuse University, Syracuse, NY 13244, USA \\ ${ }^{5}$ Jet Propulsion Laboratory, California Institute of Technology, Pasadena, CA 91106, USA \\ ${ }^{6}$ Center for Radiophysics and Space Research, Cornell University, Ithaca, New York 14853, USA \\ ${ }^{7}$ Lawrence Berkeley National Laboratory, Berkeley, CA 94720, USA \\ ${ }^{8}$ Canadian Institute for Theoretical Astrophysics, 60 St. George Street, University of Toronto, Toronto, ON M5S 3H8, Canada \\ ${ }^{9}$ Department of Physics and Astronomy, Washington State University, Pullman, Washington 99164, USA
}

(Dated: January 22, 2016)

\begin{abstract}
Gravitational waves from binary neutron star (BNS) and black-hole/neutron star (BHNS) inspirals are primary sources for detection by the Advanced Laser Interferometer Gravitational-Wave Observatory. The tidal forces acting on the neutron stars induce changes in the phase evolution of the gravitational waveform, and these changes can be used to constrain the nuclear equation of state. Current methods of generating BNS and BHNS waveforms rely on either computationally challenging full $3 \mathrm{D}$ hydrodynamical simulations or approximate analytic solutions. We introduce a new method for computing inspiral waveforms for BNS/BHNS systems by adding the post-Newtonian (PN) tidal effects to full numerical simulations of binary black holes (BBHs), effectively replacing the non-tidal terms in the PN expansion with BBH results. Comparing a waveform generated with this method against a full hydrodynamical simulation of a BNS inspiral yields a phase difference of $<1$ radian over $\sim 15$ orbits. The numerical phase accuracy required of BNS simulations to measure the accuracy of the method we present here is estimated as a function of the tidal deformability parameter $\lambda$.
\end{abstract}

Introduction. In September 2015, the Advanced Laser Interferometer Gravitational-Wave Observatory (aLIGO) began searching for gravitational waves (GWs) [1], and will be followed by advanced Virgo [2] and KAGRA [3]. The most likely GW sources for these detectors are mergers of binaries consisting of neutron stars (NSs) or black holes (BHs) [4]. If both objects are NSs (BNS), or if one is a NS and the other is a BH (a BHNS binary), then the tidal deformability of the NS will alter the GW signal in a way dependent upon the NS equation of state (EOS), allowing these observatories to constrain the EOS [5-13]. It is therefore of key importance to understand and model the influence of tidal effects on BNS and BHNS waveforms. We show here that a binary black hole (BBH) waveform can be augmented with PN tidal effects to accurately model a BNS system during the inspiral portion of the binary evolution. In principle, this method should also be applicable to BHNS systems.

BNS waveforms are typically computed using PostNewtonian (PN) methods, which are perturbative expansions in the invariant velocity $v=(M d \phi / d t)^{1 / 3}$, where $M$ is the total mass of the system and $\phi$ is the orbital phase (here we assume $c=G=1$ ). For binaries consisting of non-spinning point-particles, the expansion is known through 3.5PN order [14]. The static NS tidal effects first enter at 5PN order and depend upon the tidal deformability $\lambda_{i}[15]$. The parameter $\lambda_{i}$ measures how much each NS $i$ deforms in the presence of a tidal field, and depends on the NS mass and EOS implicitly through its dimensionless Love number $k_{2, i}$ and radius $R_{i}: \lambda_{i}=(2 / 3) k_{2, i} R_{i}^{5}$ [5]. As $v$ increases throughout the inspiral, the missing $4 \mathrm{PN}, 4.5 \mathrm{PN}$, and $5 \mathrm{PN}$ point-particle terms can result in the late portion of the PN waveform becoming inaccurate before the static tidal terms are large enough to contribute. For estimating the NS tidal deformability by using PN waveforms, the error introduced by neglecting the higher order PN terms can be as large as the statistical errors due to noise in the measured signals $[11,12,16,17]$.

Effective-one-body (EOB) models that include tidal effects [18-20] also include the merger, and provide better accuracy than $\mathrm{PN}$ by tuning higher-order vacuum terms to numerical relativity (NR) BBH waveforms. Although EOB has accurately reproduced waveforms from NR BNS simulations [20,21], here we discuss a new and different approach that holds considerable promise for modeling tidal interactions during the inspiral.

The most accurate method of computing waveforms is carrying out full NR simulations for BNS and BHNS binaries; see [10, 20-30] for recent work. However, BNS and BHNS simulations are computationally challenging, since they require solving not only the full Einstein equations but also relativistic hydrodynamics with a realistic EOS. It is unfeasible to use NR hydrodynamic simulations alone to cover the parameter space given the wide 
range of theoretically possible EOS and NS masses. In contrast, BBH systems are easier to simulate with higher accuracy. Several large catalogs of BBH simulations and resulting waveforms have been compiled [31-37].

We introduce here a method we call "PN Tidal Splicing", which generates BNS inspiral waveforms from NR $\mathrm{BBH}$ waveforms by adding tidal interactions derived in the $\mathrm{PN}$ formalism, effectively replacing the point-particle $\mathrm{PN}$ terms by the numerical BBH evolution.

We compare PN tidal splicing to NR using two simulations generated by SpEC [39], a code developed to evolve Einstein's equations and general relativistic hydrodynamics [40, 41]. The first is a new equal-mass BNS simulation with 22 orbits before merger [42], and the neutron stars were initialized with gravitational masses $m_{i} \approx 1.64 M_{\odot}$ and a polytropic EOS with $P=$ $123.6 M_{\odot}^{2} \rho^{2}$, leading to a tidal deformability of $\lambda_{i} \approx$ $5.7 \times 10^{36} \mathrm{~g} \mathrm{~cm}^{2} \mathrm{~s}^{2}$. The other is an equal-mass, nonspinning BBH simulation [43] tagged SXS:BBH:0180 in the public simulation catalog of the Simulating eXtreme Spacetimes collaboration [35]. Using tidal splicing, we add tidal terms to the $\mathrm{BBH}$ waveform in an attempt to reproduce the BNS waveform. As a test, we also subtract tidal terms from the BNS waveform in an attempt to reproduce the $\mathrm{BBH}$ waveform.

Figure 1 shows that the GW phase difference, $\left|\delta \phi_{\mathrm{GW}}\right|$, between the 'BBH+tidal' waveform and the BNS waveform is the same as the difference between the 'BNS-tidal' waveform and the BBH waveform, and both are a factor of $\sim 3$ smaller than the difference between the BNS and $\mathrm{BBH}$ waveforms throughout the inspiral. Thus we can mimic the inspiral of a full BNS simulation to within a few tenths of a radian at a fraction of the cost. For the BBH waveform, the phase error is estimated by the phase difference between the highest two resolutions. The BNS simulation is a combination of spectral and finite-volume methods with complicated convergence properties; it is unclear how to construct an accurate error measure [42]. We choose the simple prescription of plotting the phase difference between the highest two resolutions as a crude error estimate. While the BBH error estimate is small, the error estimate in the BNS simulation is as large as the tidal effects themselves. Therefore, we cannot yet fully verify the accuracy of tidal splicing until more accurate BNS simulations are available. Below (cf. Fig. 3) we will estimate the phase accuracy required of future BNS simulations for such verification.

Methods. For non-precessing binaries, the PN equations for quasicircular orbits read

$$
\begin{aligned}
& \frac{d v}{d t}=F(v), \\
& \frac{d \phi}{d t}=v^{3} / M,
\end{aligned}
$$

where $F(v)$ is the ratio of two functions, each known to finite PN order in $v$, and also depends on the binary's intrinsic parameters [44]. Different ways of evaluating these equations result in different $\mathrm{PN}$ approximants that agree to the same PN order in $v$, but diverge at higher orders. We present methods for tidal splicing using two different approximants.

TaylorT4. If $F(v)$ is expanded as a series in $v$ and then truncated to the appropriate $\mathrm{PN}$ order, then the solution is known as the TaylorT4 approximant [45]. For TaylorT4, the tidal effects manifest as additional terms in the power series for $F(v)$. Eq. (1) can be written

$$
\frac{d v}{d t}=F(v)=F_{\mathrm{pp}}(v)+F_{\mathrm{tid}}(v),
$$

where $F_{\mathrm{pp}}(v)$ are the point-particle terms, and where the additional static tidal terms $F_{\text {tid }}(v)$ are known to $6 \mathrm{PN}$ order [15].

For inspiraling PN BBHs, $F(v)$ is governed by the point-particle terms. PN tidal splicing uses $\phi(t)$ from a BBH simulation together with Eqs. (3) and (2) (with $F_{\text {tid }}(v)$ set to zero) to compute an accurate version of $F_{\mathrm{pp}}(v)$, which we will call $F_{\mathrm{NR}}(v)$. To do this, we set $\phi(t)=\phi_{\mathrm{GW}} / 2$, where $\phi_{\mathrm{GW}}$ is the $\mathrm{GW}$ phase of the $\ell=m=2$ spherical-harmonic mode of the NR waveform. Then Eq. (2) yields

$$
v(t)=\left(\frac{M}{2} \frac{d \phi_{\mathrm{GW}}}{d t}\right)^{1 / 3} .
$$

Given $v(t)$, we compute $F_{\mathrm{NR}}(v)=d v / d t$ using finite differencing. Assuming $v(t)$ is monotonic, we can write $F_{\mathrm{NR}}(v)$ as a single-valued function of $v$.

Using this $F_{\mathrm{NR}}(v)$ in place of $F_{\mathrm{pp}}(v)$ in Eq. (3), we then re-solve Eqs. (3) and (2), including the tidal terms $F_{\text {tid }}(v)$, to generate a waveform for a binary that includes tidal interactions. We express the orbital evolution of the new binary in terms of a new time coordinate $\bar{t}$. From the analytic expression for $F_{\text {tid }}(v)[15]$ and Eq. (3) we write a differential equation for $\bar{t}$ :

$$
\frac{d \bar{t}}{d v}=\frac{1}{F_{\mathrm{NR}}(v)+F_{\mathrm{tid}}(v)} .
$$

Integrating this expression and inverting yields the function $v(\bar{t})$ corresponding to the spliced waveform.

The phase of the spliced waveform, $\bar{\phi}_{\mathrm{GW}}(\bar{t})$, is computed by integrating Eq. (2):

$$
\bar{\phi}_{\mathrm{GW}}(\bar{t})=\frac{2}{M} \int_{\bar{t}_{\min }}^{\bar{t}} v(\bar{t})^{3} d \bar{t},
$$

where $\bar{t}_{\min }$ is the start of the numerical waveform.

In TaylorT4, the amplitude of the waveform is a function of $v$ only, with no explicit time dependence [46]. So here we assume that the amplitude of the original NR waveform $A_{\mathrm{NR}}(t)$ is likewise a function of $v$ only, so that we can write $A_{\mathrm{NR}}(v)=A_{\mathrm{NR}}(t(v))$. We then use $v(\bar{t})$ to express this amplitude in terms of $\bar{t}$. In 


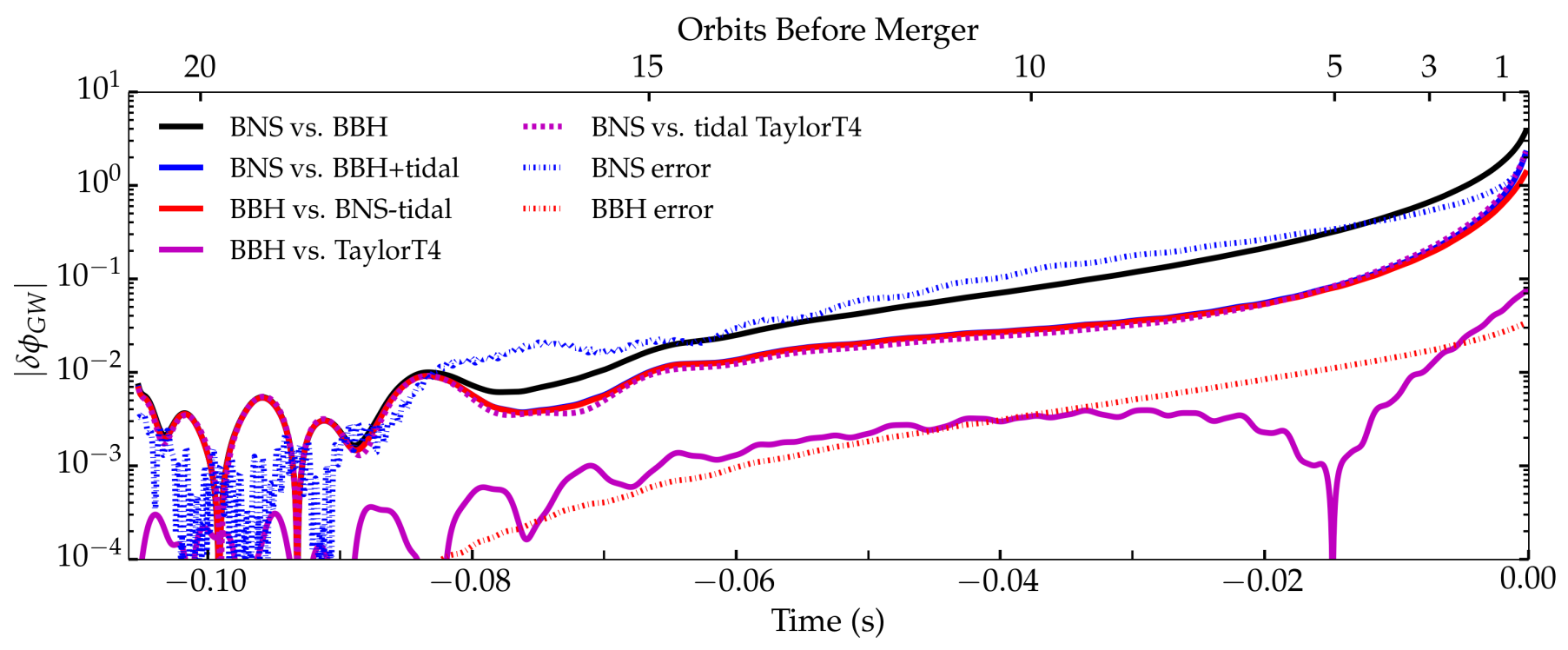

FIG. 1. Phase difference between gravitational waveforms as a function of time, for an equal-mass binary of nonspinning compact objects. Differences are shown between BNS and BBH waveforms (black), between a BBH waveform with TaylorT4 tidal terms added and a BNS waveform (blue), and between a BNS waveform with TaylorT4 tidal terms subtracted and a BBH waveform (red). The red and blue curves nearly coincide. Also shown are the phase differences between BBH and point-particle TaylorT4 waveforms (solid magenta) and between BNS and tidal TaylorT4 waveforms (dashed magenta). The numerical error in the BBH waveform (dashed red) and an estimate of the error in the BNS waveform (dashed blue) are also shown. All waveforms are aligned with the BNS waveform according to [38]; the alignment time window encompasses a $5 \%$ change around a GW frequency of $280 \mathrm{~Hz}$ for a total mass of $M=2 \times 1.64 M_{\odot}$. The blue and red curves are smaller than the black curve by a factor of $\sim 3$, demonstrating that tidal splicing can generate a BNS waveform from a BBH waveform and vice versa. The large error in the BNS waveform prevents us from fully measuring the accuracy of tidal splicing.

other words, the amplitude of the resulting waveform is $\bar{A}(\bar{t})=A_{\mathrm{NR}}(t(v(\bar{t})))$. We generate a $\mathrm{BBH}$ waveform from a BNS waveform by the same method, except we subtract instead of add $F_{\text {tid }}(v)$ in the denominator of Eq. (5).

We require $v(t)$ to be monotonic so that $F(v)$ is single-valued. To remove high-frequency numerical noise, the derivative in Eq. (4) is computed with a 3rd order Savitzky-Golay filter [47] with a window size of $\approx 48.5 \mu \mathrm{s}$. This is sufficient when adding tidal terms to the BBH waveform considered here. However, when testing our method by subtracting tidal terms from a BNS waveform, the phase of the BNS waveform considered here [42] has large enough oscillations in $v(t)$ that stronger smoothing is needed. We proceed by first subtracting the phase of the TaylorT4 waveform from that of the BNS waveform, expanding this difference in Chebyshev polynomials, truncating the Chebyshev expansion to $n=35$, and adding back the phase of the TaylorT4 waveform. We find that the difference between the smoothed and unsmoothed phase of the BNS waveform is less than $3 \times 10^{-3}$ radians.

As discussed above, Figure 1 displays the phase differences between NR and tidally-spliced TaylorT4 waveforms. We now examine how well pure PN waveforms agree with NR waveforms. The magenta solid and dashed curves in Fig. 1 show phase differences between TaylorT4 and BNS or BBH waveforms. The point-particle Tay-
lorT4 waveform does an excellent job of reproducing the phase evolution of the BBH waveform, about at the level of the BBH numerical error. However, while TaylorT4 is surprisingly accurate in the inspiral for equal-mass, nonspinning systems [45, 48], this does not hold true in general [49-51]. Tidal splicing should be applicable to an arbitrary BNS/BHNS system with spins and/or unequal masses, where there may not be an accurate PN approximant. References $[11,12]$ showed that uncertainties in the PN waveforms are one of the largest sources of error for tidal parameter estimation, and conclude that more accurate waveforms are needed.

TaylorF2. If Eqs. (1) and (2) are instead converted to the frequency domain (FD) using the stationary phase approximation before expanding the series, the approximant is called TaylorF2 [52]. TaylorF2 waveforms are expressed in the FD, and can be written

$$
\tilde{h}(f)=\tilde{A}(f) \exp (i \tilde{\Psi}(f))
$$

where $\tilde{A}(f)$ is real and $\tilde{\Psi}(f)$ is the Fourier phase as a function of the GW frequency $f=v^{3} /(\pi M)$. For pointparticles, $\tilde{\Psi}(f)=\tilde{\Psi}_{\mathrm{pp}}(f)$ is known for non-spinning systems to $3.5 \mathrm{PN}$ order $[52,53]$. For tidally deformable objects, we write $\tilde{\Psi}(f)=\tilde{\Psi}_{\mathrm{pp}}(f)+\tilde{\Psi}_{\text {tid }}(f)$, where $\tilde{\Psi}_{\text {tid }}(f)$ has been calculated up to 7.5PN order, with the exception of a few unknown constants [7, 19]. Here we include 


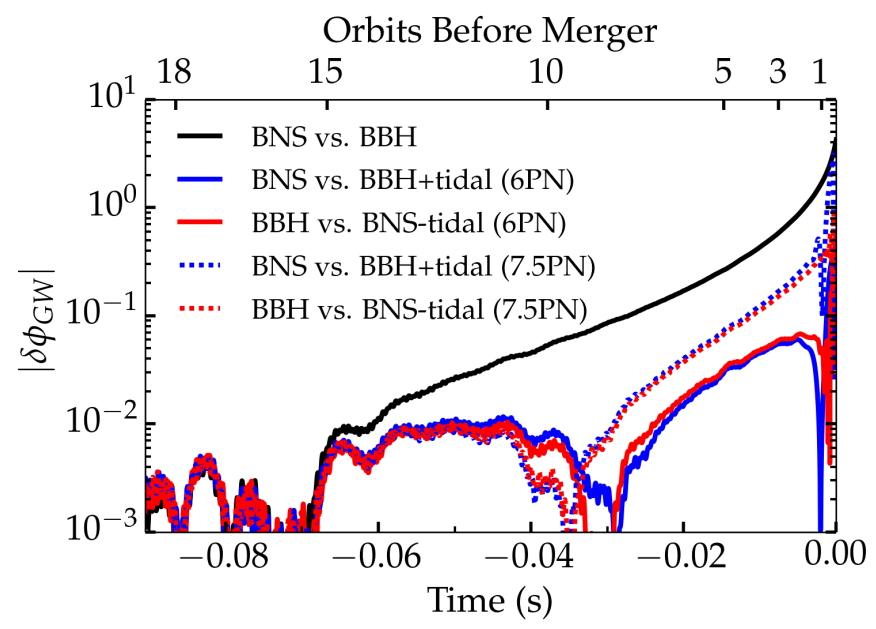

FIG. 2. The phase difference $\left|\delta \phi_{\mathrm{GW}}(t)\right|$ as a function of time for waveforms spliced with TaylorF2. Differences are shown between a BNS and a BBH waveform (black), between a 'BBH+tidal' and a BNS waveform (blue), and between a 'BNS-tidal' waveform and a $\mathrm{BBH}$ waveform (red) at the $6 \mathrm{PN}$ (solid) and 7.5PN (dot-dashed) orders. Only the time after the windowing function is shown here, resulting in a shorter time axis here than in Fig. 1. The late-time noise is an artifact caused by inverse Fourier transforming the unphysical high-frequency behavior of $\tilde{\Psi}_{\text {tid }}(f)$. At both PN orders, tidal splicing can generate a BNS waveform from a $\mathrm{BBH}$ waveform and vice versa.

both $6 \mathrm{PN}$ tidal effects and 7.5PN tidal effects, setting the unknown constants to 0 as was done in [13].

To add the static tidal terms to an existing $\mathrm{BBH}$ waveform, first the Fourier transform of the waveform $\tilde{h}_{\mathrm{NR}}(f)$ is computed. The early portion of the waveform is windowed using a Planck-taper [54] while the merger and ringdown provide a natural windowing for the late portion. We then compute $\tilde{\Psi}_{\mathrm{NR}}(f)$ and $\tilde{A}_{\mathrm{NR}}(f)$ by decomposing according to Eq. (7). The spliced Fourier phase is then $\tilde{\Psi}(f)=\tilde{\Psi}_{\mathrm{NR}}(f)+\tilde{\Psi}_{\mathrm{tid}}(f)$. Because the known tidal terms do not affect the amplitude $\tilde{A}_{\mathrm{NR}}(f)$, the new waveform is then

$$
\tilde{h}(f)=\tilde{A}_{\mathrm{NR}}(f) \exp \left(i\left[\tilde{\Psi}_{\mathrm{NR}}(f)+\tilde{\Psi}_{\mathrm{tid}}(f)\right]\right) .
$$

No smoothing of the numerical waveforms is needed for TaylorF2 splicing, unlike the TaylorT4 case.

Since the PN approximation breaks down for high frequencies, we impose a high frequency cutoff which we choose to be $f_{\mathrm{ISCO}}=1 /\left(6^{3 / 2} \pi M\right)=1338 \mathrm{~Hz}$, the GW frequency corresponding to the innermost stable circular orbit of a Schwarzschild black hole of mass equal to the total mass of the system. It has been shown that for BNS systems, $f_{\mathrm{ISCO}}$ is approximately the merger frequency [55]. The starting frequency of the NR BNS waveform after windowing is $\sim 285 \mathrm{~Hz}$.

We estimate the error of the spliced waveforms by analyzing the phase differences in time domain after taking

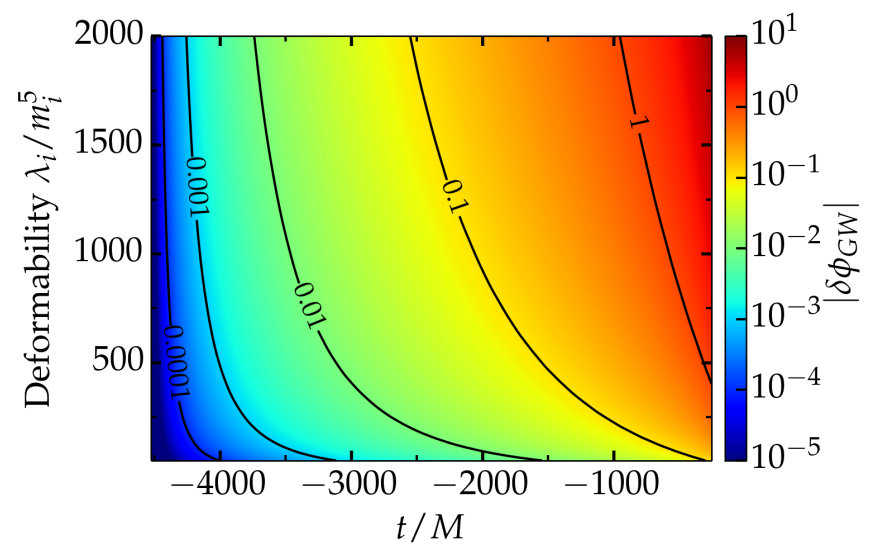

FIG. 3. Phase difference between equal-mass, nonspinning $\mathrm{BBH}$ and 'BBH+tidal' waveforms. Each horizontal slice through this plot shows the phase difference as a function of time for a particular dimensionless deformability $\lambda_{i} / m_{i}^{5}$. For our BNS simulation, $\lambda_{i} / m_{i}^{5} \approx 453$. Contours show selected values of the phase differerence. A BNS simulation starting at dimensionless time $t / M \approx-4500$ would need phase errors smaller than the values shown here in order to measure tidal effects. Even more accurate BNS simulations would be needed to measure the accuracy of the tidal splicing method.

the inverse Fourier transform. To avoid jump discontinuities in the Fourier phase, we roll off the effect of $\tilde{\Psi}_{\text {tid }}(f)$ from $f_{\text {ISCO }}$ to $2 \times f_{\text {ISCO }}$ with a cosine window. While this will contaminate the higher frequency content, this should allow the lower frequencies of the inspiral to be mostly unaffected. After the inverse Fourier transform, the time domain waveforms are aligned in a $10 \%$ region around $300 \mathrm{~Hz}$. The phase differences are shown in Fig. 2 and are similar to Fig. 1. With the exception of the last $\sim 3 \mathrm{~ms}$ of the waveforms, which are affected by the high frequency contamination, all of the spliced waveforms maintain phase differences under 0.1 radians during most of the inspiral, below the difference between the BNS and BBH waveforms. It is not clear why the $6 \mathrm{PN}$ terms approximate the tidal effects better than the $7.5 \mathrm{PN}$ terms; it may be because we zero the unknown constants in the 7.5PN expression.

Discussion. We have shown that PN tidal splicing of $\mathrm{BBH}$ waveforms can produce inspiral waveforms for nonspinning BNS systems. This method should easily generalize to objects with spins and to BHNS systems. Once a particular mass ratio and spin configuration $\mathrm{BBH}$ waveform is generated, it should be easy to produce BNS/BHNS waveforms via PN tidal splicing for any EOS simply by adjusting the tidal parameters $\lambda_{i}$, allowing the entire tidal parameter space for inspiral waveforms to be spanned.

The accuracy of this method is limited by that of the PN tidal terms. In particular, additional finite size effects not captured by the current static tidal PN terms can influence waveform amplitude and phase, and dy- 
namical tidal effects can also contribute to the phase evolution [56], especially as the NS's approach merger. This method in principle can be improved with better PN tidal terms. Unfortunately, it is currently difficult to fully measure the accuracy of tidal splicing until higheraccuracy many-orbit BNS simulations are available for multiple masses and EOS.

Figure 3 estimates the accuracy needed for equal mass, nonspinning BNS simulations to see the tidal effects on the inspiral phase of the waveform. Even smaller BNS errors would be necessary to constrain the accuracy of tidal splicing. We chose the start time in Fig. 3 so that the inspiral spans a large enough frequency range for aLIGO to recover $97 \%$ of the information about $\lambda_{i}$, according to the analysis presented in Fig. 3 of [7]. We assume $M=2.8 M_{\odot}$ (corresponding to a prototypical NS mass of $1.4 M_{\odot}$ ) and an upper frequency cutoff of $f_{\text {ISCO }}$.

An alternative to computing tidal terms to a higher $\mathrm{PN}$ order is to resum them in some way, as is done in $[20,21$, 57 in the context of EOB. It is not clear how to do this with tidal splicing.

Additionally, the merger/ringdown cannot be modeled with splicing alone, especially for BNS mergers and BHNS systems that undergo tidal disruption. One possibility is to combine an analytic waveform in the very early inspiral with a spliced $\mathrm{BBH}$ waveform in the mid to late inspiral and then with a waveform from a full hydrodynamical simulation for the merger and ringdown, to create a 'tribridized' waveform. This might reduce the need for expensive hydrodynamical simulations lasting many orbits. If necessary, surrogate models [43, 58, 59] that cover the parameter space including the EOS can be forged from spliced BBH waveforms.

Acknowledgements. We thank Harald Pfeiffer and Sanjay Reddy for helpful discussions. This work was supported in part by the Sherman Fairchild Foundation and NSF grants PHY-1404569 and AST-1333520 at Caltech, NSF grant AST-1333142 at Syracuse University, the Sherman Fairchild Foundation and NSF Grants PHY1306125 and AST-1333129 at Cornell University and by NASA through Einstein Postdoctoral Fellowship grant numbered PF4-150122 awarded by the Chandra X-ray Center, which is operated by the Smithsonian Astrophysical Observatory for NASA under contract NAS8-03060. Computations were performed on the Zwicky cluster at Caltech, which is supported by the Sherman Fairchild Foundation and by NSF award PHY-0960291; on the NSF XSEDE network under grant TG-PHY990007N; on the NSF/NCSA Blue Waters at the University of Illinois with allocation jr6 under NSF PRAC Award ACI1440083; and on the GPC supercomputer at the SciNet HPC Consortium [60]; SciNet is funded by: the Canada Foundation for Innovation (CFI) under the auspices of Compute Canada; the Government of Ontario; Ontario Research Fund (ORF) - Research Excellence; and the University of Toronto.
[1] J. Aasi et al. (LIGO Scientific Collaboration), Class. Quantum Grav. 32, 074001 (2015), arXiv:1411.4547 [grqc].

[2] F. Acernese et al. (Virgo Collaboration), Class. Quantum Grav. 32, 024001 (2015), arXiv:1408.3978 [gr-qc].

[3] Y. Aso, Y. Michimura, K. Somiya, M. Ando, O. Miyakawa, T. Sekiguchi, D. Tatsumi, and H. Yamamoto (The KAGRA Collaboration), Phys. Rev. D 88, 043007 (2013), arXiv:1306.6747 [gr-qc].

[4] J. Abadie et al. (LIGO Scientific Collaboration), Class. Quantum Grav. 27, 173001 (2010), arXiv:1003.2480 [grqc].

[5] É. É. Flanagan and T. Hinderer, Phys. Rev. D 77, 021502 (2008), arXiv:0709.1915.

[6] T. Hinderer, B. D. Lackey, R. N. Lang, and J. S. Read, Phys. Rev. D 81, 123016 (2010), arXiv:0911.3535 [astroph.HE].

[7] T. Damour, A. Nagar, and L. Villain, Phys. Rev. D 85, 123007 (2012), arXiv:1203.4352 [gr-qc].

[8] W. Del Pozzo, T. G. F. Li, M. Agathos, C. Van Den Broeck, and S. Vitale, Phys. Rev. Lett. 111, 071101 (2013), arXiv:1307.8338 [gr-qc].

[9] A. Maselli, L. Gualtieri, and V. Ferrari, Phys.Rev. D88, 104040 (2013), arXiv:1310.5381 [gr-qc].

[10] J. S. Read, L. Baiotti, J. D. E. Creighton, J. L. Friedman, B. Giacomazzo, K. Kyutoku, C. Markakis, L. Rezzolla, M. Shibata, and K. Taniguchi, Phys. Rev. D 88, 044042 (2013), arXiv:1306.4065 [gr-qc].

[11] L. Wade, J. D. E. Creighton, E. Ochsner, B. D. Lackey, B. F. Farr, et al., Phys.Rev. D89, 103012 (2014), arXiv:1402.5156 [gr-qc].

[12] B. D. Lackey and L. Wade, Phys. Rev. D 91, 043002 (2014), arXiv:1410.8866 [gr-qc].

[13] M. Agathos, J. Meidan, W. D. Pozzo, T. G. F. Li, M. Tompitak, J. Veitch, S. Vitale, and C. V. D. Broeck, prd 92, 023012 (2015), arXiv:1503.05405 [gr-qc].

[14] L. Blanchet, T. Damour, G. Esposito-Farèse, and B. R. Iyer, Phys. Rev. D 71, 124004 (2005).

[15] J. Vines, É. É. Flanagan, and T. Hinderer, Phys. Rev. D 83, 084051 (2011), arXiv:1101.1673 [gr-qc].

[16] M. Favata, Phys.Rev.Lett. 112, 101101 (2014), arXiv:1310.8288 [gr-qc].

[17] K. Yagi and N. Yunes, Phys.Rev. D89, 021303 (2014), arXiv:1310.8358 [gr-qc].

[18] T. Damour and A. Nagar, Phys. Rev. D 81, 084016 (2010), arXiv:0911.5041 [gr-qc].

[19] D. Bini, T. Damour, and G. Faye, Phys. Rev. D 85, 124034 (2012), arXiv:1202.3565 [gr-qc].

[20] S. Bernuzzi, A. Nagar, T. Dietrich, and T. Damour, Phys.Rev.Lett. 114, 161103 (2015), arXiv:1412.4553 [grqc].

[21] K. Hotokezaka, K. Kyutoku, H. Okawa, and M. Shibata, Phys. Rev. D91, 064060 (2015), arXiv:1502.03457 [grqc].

[22] D. Radice, L. Rezzolla, and F. Galeazzi, Mon. Not. Roy. Astron. Soc. 437, L46 (2014), arXiv:1306.6052 [gr-qc].

[23] K. Hotokezaka, K. Kyutoku, and M. Shibata, Phys.Rev. D87, 044001 (2013), arXiv:1301.3555 [gr-qc].

[24] S. Bernuzzi, A. Nagar, M. Thierfelder, and B. Brügmann, Phys. Rev. D 86, 044030 (2012), 
arXiv:1205.3403 [gr-qc].

[25] S. Bernuzzi, M. Thierfelder, and B. Bruegmann, Phys. Rev. D 85, 104030 (2012), arXiv:1109.3611 [gr-qc].

[26] L. Baiotti, T. Damour, B. Giacomazzo, A. Nagar, and L. Rezzolla, Phys. Rev. D 84, 024017 (2011), arXiv:1103.3874 [gr-qc].

[27] L. Baiotti, T. Damour, B. Giacomazzo, A. Nagar, and L. Rezzolla, Phys. Rev. Lett. 105, 261101 (2010), arXiv:1009.0521 [gr-qc].

[28] K. Kawaguchi, K. Kyutoku, H. Nakano, H. Okawa, M. Shibata, and K. Taniguchi, Phys. Rev. D 92, 024014 (2015), arXiv:1506.05473 [astro-ph.HE].

[29] F. Pannarale, E. Berti, K. Kyutoku, and M. Shibata, Phys.Rev. D88, 084011 (2013), arXiv:1307.5111 [gr-qc].

[30] F. Foucart, L. Buchman, M. D. Duez, M. Grudich, L. E. Kidder, I. MacDonald, A. Mroue, H. P. Pfeiffer, M. A. Scheel, and B. Szilagyi, Phys. Rev. D 88, 064017 (2013), arXiv:1307.7685 [gr-qc].

[31] B. Aylott, J. G. Baker, W. D. Boggs, M. Boyle, P. R. Brady, et al., Class. Quantum Grav. 26, 165008 (2009), arXiv:0901.4399 [gr-qc].

[32] P. Ajith, M. Boyle, D. A. Brown, B. Brugmann, L. T. Buchman, et al., Class. Quantum Grav. 29, 124001 (2012).

[33] L. Pekowsky, R. O'Shaughnessy, J. Healy, and D. Shoemaker, Phys. Rev. D 88, 024040 (2013), arXiv:1304.3176 [gr-qc].

[34] I. Hinder et al. (The NRAR Collaboration), Class. Quantum Grav. 31, 025012 (2014), arXiv:1307.5307 [gr-qc].

[35] http://www.black-holes.org/waveforms.

[36] J. Clark, L. Cadonati, J. Healy, I. Heng, J. Logue, N. Mangini, L. London, L. Pekowsky, and D. Shoemaker, in Gravitational Wave Astrophysics, Astrophysics and Space Science Proceedings, Vol. 40, edited by C. F. Sopuerta (Springer International Publishing, 2015) pp. 281-287, arXiv:1406.5426 [gr-qc].

[37] J. Healy, P. Laguna, and D. Shoemaker, Class. Quantum Grav. 31, 212001 (2014), arXiv:1407.5989 [gr-qc].

[38] M. Boyle, A. Buonanno, L. E. Kidder, A. H. Mroué, Y. Pan, et al., Phys. Rev. D 78, 104020 (2008), arXiv:0804.4184 [gr-qc].

[39] http://www.black-holes.org/SpEC.html.

[40] M. D. Duez, F. Foucart, L. E. Kidder, H. P. Pfeiffer, M. A. Scheel, and S. A. Teukolsky, Phys. Rev. D 78, 104015 (2008), arXiv:0809.0002 [gr-qc].

[41] F. Foucart, M. B. Deaton, M. D. Duez, L. E. Kidder, I. MacDonald, C. D. Ott, H. P. Pfeiffer, M. A. Scheel, B. Szilagyi, and S. A. Teukolsky, Phys. Rev. D 87, 084006 (2013), arXiv:1212.4810 [gr-qc].

[42] R. Haas, B. Szilagyi, J. D. Kaplan, C. D. Ott, J. Lippuner, M. A. Scheel, K. Barkett, C. D. Muhlberger,
F. Foucart, and M. D. Duez, in preparation (2015).

[43] J. Blackman, S. E. Field, C. R. Galley, B. Szilágyi, M. A. Scheel, M. Tiglio, and D. A. Hemberger, Phys. Rev. Lett. 115, 121102 (2015), arXiv:1502.07758 [gr-qc].

[44] L. Blanchet, Living Rev.Rel. 17, 2 (2014).

[45] M. Boyle, D. A. Brown, L. E. Kidder, A. H. Mroué, H. P. Pfeiffer, M. A. Scheel, G. B. Cook, and S. A. Teukolsky, Phys. Rev. D 76, 124038 (2007), arXiv:0710.0158 [gr-qc].

[46] L. Blanchet, G. Faye, B. R. Iyer, and S. Sinha, Class. Quantum Grav. 25, 165003 (2008), arXiv:0802.1249 [grqc].

[47] W. H. Press, S. A. Teukolsky, W. T. Vetterling, and B. P. Flannery, Numerical Recipes: The Art of Scientific Computing, 3rd ed. (Cambridge University Press, 2007).

[48] I. MacDonald, S. Nissanke, and H. P. Pfeiffer, Class. Quantum Grav. 28, 134002 (2011), arXiv:1102.5128 [gr$\mathrm{qc}]$.

[49] M. Hannam, S. Husa, B. Brügmann, and A. Gopakumar, Phys. Rev. D 78, 104007 (2008).

[50] M. Hannam, S. Husa, F. Ohme, D. Muller, and B. Brugmann, Phys.Rev. D82, 124008 (2010), arXiv:1007.4789 [gr-qc].

[51] B. Szilagyi, J. Blackman, A. Buonanno, A. Taracchini, H. P. Pfeiffer, et al., Phys. Rev. Lett. 115, 031102 (2015), arXiv:1502.04953 [gr-qc].

[52] T. Damour, B. R. Iyer, and B. Sathyaprakash, Phys.Rev. D63, 044023 (2001), arXiv:gr-qc/0010009 [gr-qc].

[53] T. Damour, B. R. Iyer, and B. S. Sathyaprakash, Phys. Rev. D 66, 027502 (2002), erratum: [61].

[54] D. McKechan, C. Robinson, and B. Sathyaprakash, Class. Quantum Grav. 27, 084020 (2010), arXiv:1003.2939 [gr-qc].

[55] S. Bernuzzi, A. Nagar, S. Balmelli, T. Dietrich, and M. Ujevic, Phys.Rev.Lett. 112, 201101 (2014), arXiv:1402.6244 [gr-qc].

[56] T. Hinderer, A. Taracchini, F. Foucart, A. Buonanno, J. Steinhoff, M. Duez, L. E. Kidder, H. P. Pfeiffer, M. A. Scheel, B. Szilagyi, K. Hotokezaka, K. Kyutoku, M. Shibata, and C. W. Carpenter, in preparation (2016).

[57] D. Bini and T. Damour, Phys. Rev. D 90, 124037 (2014), arXiv:1409.6933 [gr-qc].

[58] S. E. Field, C. R. Galley, J. S. Hesthaven, J. Kaye, and M. Tiglio, Phys. Rev. X 4, 031006 (2014), arXiv:1308.3565 [gr-qc].

[59] M. Pürrer, Class. Quantum Grav. 31, 195010 (2014), arXiv:1402.4146 [gr-qc].

[60] C. Loken, D. Gruner, L. Groer, R. Peltier, N. Bunn, M. Craig, T. Henriques, J. Dempsey, C.-H. Yu, J. Chen, L. J. Dursi, J. Chong, S. Northrup, J. Pinto, N. Knecht, and R. V. Zon, J. Phys.: Conf. Ser. 256, 012026 (2010).

[61] T. Damour, B. R. Iyer, and B. S. Sathyaprakash, Phys. Rev. D 72, 029901 (2005). 